\title{
SOME EXTENSIONS OF UNIVALENT CONDITIONS FOR CERTAIN INTEGRAL OPERATOR
}

\author{
DANIEL BREAZ AND SHIGEYOSHI OWA
}

Abstract. Three subclasses $\mathcal{S}(p), \mathcal{T}_{2}$ and $\mathcal{T}_{2, \mu}$ of analytic functions $f(z)$ in the open unit disk $\mathbb{U}$ are introduced. The object of the present paper is to discuss some extensions of univalent conditions for an integral operator $F_{\alpha, \beta}(z)$ of $f(z)$ belonging to the classes $\mathcal{S}(p), \mathcal{T}_{2}$ and $\mathcal{T}_{2, \mu}$.

Mathematics subject classification (2000): 30C45.

Key words and phrases: univalent, integral operator, Schwarz lemma.

\section{REFERENCES}

[1] D. BREAZ, N. BREAZ, The univalent conditions for an integral operator on the classes $\mathcal{S}(p)$ and $\mathcal{T}_{2}$, J. Approx. Theory Appl., 1, (2005), 93-98.

[2] N. PASCU, An improvement of Becker's univalence criterion, Proceedings of the Commemorative Session Simion Stoilow, Brasov, (1987), 43-48.

[3] V. SingH, On class of univalent functions, Internat. J. Math. Math. Sci. 23, (2000), 855-857. 